ANNALES

POLONICI MATHEMATICI

XLI (1983)

\title{
On the coefficients of some classes of starlike functions
}

\author{
by Ryszard Mazur (Kielce)
}

\begin{abstract}
Let $\Omega$ be the class of functions $\omega, \omega(0)=0,|\omega(z)|<1$, holomorphic in the unit disc $K$; let $a$ and $b$ be arbitrary fixed numbers, $-1 \leqslant b<a \leqslant 1, j(a, b)-$ the class of functions $P, P(0)=1$, holomorphic in $K$, such that $P \in(),(a, b)$ iff $P(z)=(1+a \omega(z))(1+b \omega(z))^{-1}$ for some function $\omega \in \Omega$ and every $z$ in $K$. Let $S^{*}(a, b)$ denote the class of functions $f, f(0)=0, f^{\prime}(0)=1$, holomorphic in $K$, such that $f \in S^{*}(a, b)$ ifl $z f^{\prime}(z)(f(z))^{-1}=P(z)$ for some $\left.P \in\right\} u(a, b) ; \Sigma^{*}(a, b)$ - the class of meromorphic functions of the form $F(z)=\frac{1}{z}+\sum_{n=0}^{x} a_{n} z^{n}$ and satisfying the condition: $z F^{\prime}(z)(F(z))^{-1}=P(z), P \in \rho(a, b)$.

The author obtains sharp estimates of the coefficients of functions of the families $S^{*}(a, b)$ and $\Sigma^{*}(a, b)$.
\end{abstract}

1. Introduction. Let $\Omega$ be the family of all functions of the form

$$
\omega(z)=c_{1} z+c_{2} z^{2}+\ldots
$$

which are holomorphic in the unit disc $K=\{z:|z|<1\}$ and satisfy the condition $|\omega(z)|<1$ for $z \in K$.

Janowski introduced (cf. [2]) a class $\wp(a, b)$ of holomorphic functions

$$
P(z)=1+p_{1} z+p_{2} z^{2}+\ldots
$$

in the unit disc; by definition, $p$ is in $\zeta(a, b)$ if and only if

$$
P(z)=\frac{1+a \omega(z)}{1+b \omega(z)}, \quad z \in K,
$$

for some function $\omega \in \Omega ; a, b$ are arbitrarily fixed numbers that satisfy the condition $-1 \leqslant b<a \leqslant 1$. It is easy to notice that $(\rho)(a, b) \subset \xi)$ and $(),(1,-1) \equiv \wp$, where $\wp$ is the well-known family of functions of Carathéodory type.

Let us derrote by $S^{*}(a, b)$ (cf. [2]) the class of functions

$$
f(z)=z+a_{2} z^{2}+\ldots
$$

holomorphic in $K$, defined by the condition: $f$ is in $S^{*}(a, b)$ if and only if 
there exists a function $P \in \varphi(a, b)$ such that

$$
\frac{z f^{\prime}(z)}{f(z)}=P(z), \quad z \in K
$$

Since $\varphi(a, b) \subset \rho$ and $\varphi(1,-1) \equiv \xi$, we have $S^{*}(a, b) \subset S^{*}$ and $S^{*}(1,-1) \equiv S^{*}$, where $S^{*}$ is the well-known class of starlike functions.

Moreover, let $\Sigma^{*}(a, b)$ denote the family of meromorphic functions

$$
F(z)=\frac{1}{z}+a_{0}+a_{1} z+a_{2} z^{2}+\ldots
$$

in the unit disc such that

$$
\frac{-z F^{\prime}(z)}{F(z)}=P(z), \quad z \in K
$$

for some function $P$ in $\wp(a, b)$.

It is not difficult to see that $\Sigma^{*}(a, b) \subset \Sigma^{*}$ and $\Sigma^{*}(1,-1) \equiv \Sigma^{*}$, where $\Sigma^{*}$ is the class of starlike meromorphic functions.

In this paper we give estimates of the coefficients of the families $S^{*}(a, b)$ and $\Sigma^{*}(a, b)$.

Our results contain those of Clunie [1], Janowski [3], Kaczmarski [4], Plaskota [5], Pomerenke [6], Robertson [7], and Wieczorek [8], as particular cases.

2. Estimates of the coefficients of functions of class $S^{*}(a, b)$. We shall prove the following result.

THEOREM 1. If $f \in S^{*}(a, b)$ and $-1 \leqslant b<a \leqslant 1$, then

$$
\left|a_{n}\right| \leqslant \frac{1}{(n-1) !} \prod_{k=1}^{n-1}|a-k b| \quad \text { for } \quad n=2,3, \ldots, p
$$

and

$$
\left|a_{n}\right| \leqslant \frac{1}{(n-1)(p-2) !} \prod_{k=1}^{p-1}|a-k b| \quad \text { for } \quad n=p+1, \ldots,
$$

where $p \in\left\langle\frac{1+a}{1+b}, \frac{2+a+b}{1+b}\right)$ is a natural number. If $b=-1$, then

$$
\left|a_{n}\right| \leqslant \frac{1}{(n-1) !} \prod_{k=1}^{n-1}(a+k) \quad \text { for } \quad n=2,3, \ldots
$$

The bounds (2.1) and (2.3) are attained by the function

$$
f(z)=\left\{\begin{array}{lll}
z(1+\varepsilon b z)^{(a-b) / b} & \text { for } & b \neq 0, \\
z_{1} \exp (a \varepsilon z) & \text { for } & b=0,
\end{array} \quad|\varepsilon|=1 .\right.
$$


Proof. If $f \in S^{*}(a, b)$, then

$$
\frac{z f^{\prime}(z)}{f(z)}=\frac{1+a \omega(z)}{1+b \omega(z)}, \quad z \in K
$$

for some function $\omega \in \Omega$.

From (2.5) it follows that

$$
z f^{\prime}(z)-f(z)=\left(a f(z)-b z f^{\prime}(z)\right) \omega(z)
$$

hence

$$
\sum_{k=1}^{x}(k-1) a_{k} z^{k}=\omega(z) \cdot \sum_{k=1}^{x}(a-k b) a_{k} z^{k}
$$

and it is easy to see that $\left|a_{2}\right| \leqslant a-b$.

Thus estimate (2.1) holds true for $n=2$. Now suppose that $n \geqslant 2$. Let us write equality (2.6) as follows:

$$
\sum_{k=1}^{n}(k-1) a_{k} z^{k}+\sum_{k=n+1}^{\infty} d_{k} z^{k}=\omega(z) \sum_{k=1}^{n-1}(a-k b) a_{k} z^{k}
$$

where the series $\sum_{k=n+1}^{x_{i}} d_{k} z^{k}$ is convergent in the unit disc. By using the method of Clunie's (cf. [1]) we get:

$$
\left|\sum_{k=1}^{n}(k-1) a_{k} z^{k}+\sum_{k=n+1}^{\infty} d_{k} z^{k}\right|<\left|\sum_{k=1}^{n-1}(a-k b) a_{k} z^{k}\right| .
$$

Hence, putting $z=r e^{i t}, 0<r<1,0 \leqslant t<2 \pi$, we have

$$
\frac{1}{2 \pi} \int_{0}^{2 \pi}\left|\sum_{k=1}^{n}(k-1) a_{k} r^{k} e^{i t k}+\sum_{k=n+1}^{x_{J}} d_{k} r^{k} e^{i k k}\right|^{2} d t \leqslant \frac{1}{2 \pi} \int_{0}^{2 \pi}\left|\sum_{k=1}^{n-1}(a-k b) a_{k} r^{k} e^{i t k}\right|^{2} d t .
$$

Integrating we get

$$
\sum_{k=1}^{n}(k-1)^{2}\left|a_{k}\right|^{2} r^{2 k}+\sum_{k=n+1}^{\infty}\left|d_{k}\right|^{2} r^{2 k} \leqslant \sum_{k=1}^{n-1}(a-k b)^{2}\left|a_{k}\right|^{2} r^{2 k}
$$

In particular, from (2.8) follows

$$
\sum_{k=1}^{n}(k-1)\left|a_{k}\right|^{2} r^{2 k} \leqslant \sum_{k=1}^{n-1}(a-k b)^{2}\left|a_{k}\right|^{2} r^{2 k}
$$

Passing in (2.9) to the limit as $r \rightarrow 1$ we obtain

$$
\sum_{k=1}^{n}(k-1)^{2}\left|a_{k}\right|^{2} \leqslant \sum_{k=1}^{n-1}(a-k b)^{2}\left|a_{k}\right|^{2} .
$$


Thus

$$
(n-1)^{2}\left|a_{n}\right|^{2} \leqslant \sum_{k=1}^{n-1}\left((a-k b)^{2}-(k-1)^{2}\right)\left|a_{k}\right|^{2} .
$$

We easily observe that $(a-(n-1) b)^{2}-(n-2)^{2} \geqslant 0$ iff $n \leqslant p$ and $-1<b$ $<a \leqslant 1$; moreover, if $b=-1$, then $(a+n-1)^{2}-(n-2)^{2} \geqslant 0$ for $n=2,3, \ldots$ and every $a \in(-1,1\rangle$. Estimates (2.1), (2.2) and (2.3) are obtained by induction. The function $f$ defined by formula (2.4) belongs to the family $S^{*}(a, b)$. Let $a_{n}^{*}$ denote the coefficient at $z^{n}$ in the power series representing the function (2.4). Then we have

$$
a_{n}^{*}=\frac{\varepsilon^{n-1}}{(n-1) !} \prod_{k=1}^{n-1}(a-k b)
$$

thus the estimates (2.1) and (2.3) are sharp. Q.E.D.

Theorem 1 implies the following corollaries:

COROLlary 1. The values of the function $f \in S^{*}(a, b)$ include the disc

$$
|w|<\frac{1}{2+a-b}
$$

In fact, let $f \in S^{*}(a, b)$ and $f(z) \neq w_{0}$ for every $z \in K$. Then the function

$$
g(z)=\frac{f(z)}{1-f(z) / w_{0}}=z+\left(a_{2}+1 / w_{0}\right) z^{2}+\ldots
$$

belongs to the family $S$. Thus $\left|a_{2}+1 / w_{0}\right| \leqslant 2$. Since $\left|a_{2}\right| \leqslant a-b$, then $(2.10)$ follows.

By specifying the values of the parameters appearing in Theorem 1 we obtain some interesting particular cases.

Corollary 2. By putting $a=1-2 \alpha, b=-1$ in (2.3) we obtain the result of [7] for starlike functions of order $\alpha$.

COROLlaRY 3. For $a=1$ and $b=\frac{1}{M}-1, M \geqslant 1$, we obtain the result of Janowski (cf. [3]).

Corollary 4. By substituting $b=-a, 0<a<1$, in (2.1), (2.2) and (2.10) we get corresponding results obtained in [8].

COROLlary 5. If $a=1-\lambda-\lambda m, b=-m$, then we obtain the result of [5].

Corollary 6. By applying Theorem 1 to the case where $a=1$ and $b=-1$, we obtain $\left|a_{n}\right| \leqslant n$ for starlike functions of order 0 .

3. Estimates for coefficients of functions of class $\Sigma^{*}(a, b)$. Now we shall find estimates for coefficients of functions belonging to the family $\Sigma^{*}(a, b)$. 
ThEOREM 2. If $F \in \Sigma^{*}(a, b)$, then

$$
\left|a_{n}\right| \leqslant \frac{a-b}{n+1} \quad \text { for } n=0,1,2, \ldots
$$

The bound is attained by the function

$$
F^{*}(z)= \begin{cases}\frac{1}{z}\left(1+b z^{n+1}\right)^{(b-a) / b(n+1)} & \text { for } b \neq 0, \\ \frac{1}{z} \exp \left(\frac{-a}{n+1} z^{n+1}\right) & \text { for } b=0 .\end{cases}
$$

Proof. It follows from the definitions of the families $\Sigma^{*}(a, b)$ and $\wp(a, b)$ that $F \in \Sigma^{*}(a, b)$ if and only if

$$
-\frac{z F^{\prime}(z)}{F(z)}=\frac{1+a \omega(z)}{1+b \omega(z)}, \quad z \in K
$$

for some function $\omega \in \Omega$.

From (3.3) and (1.6) we have

$$
\sum_{k=0}^{\infty}(k+1) a_{k} z^{k+1}=-\left(a-b+\sum_{k=0}^{\infty}(a+k b) a_{k} z^{k+1}\right) \omega(z) .
$$

Hence and from (1.1) we have

$$
\left|a_{0}\right| \leqslant a-b, \quad\left|a_{1}\right| \leqslant(a-b) / 2 .
$$

Let $n \geqslant 2$. By applying the method of Clunie [1] we finally obtain the inequality

$$
(n+1)^{2}\left|a_{n}\right|^{2} \leqslant(a-b)^{2}+\sum_{k=0}^{n-1}\left((a+k b)^{2}-(k+1)^{2}\right)\left|a_{k}\right|^{2} .
$$

Since $(a l+l b)^{2}-(l+1)^{2} \leqslant 0$ for $l=0,1,2, \ldots$ and $-1 \leqslant b<a \leqslant 1$, we have

$$
(n+1)^{2}\left|a_{n}\right|^{2} \leqslant(a-b)^{2} \text {. }
$$

From inequalities (3.5) and (3.7) we conclude that the estimate (3.1) holds true for $n=0,1,2, \ldots$

The function $F$ defined by formula (3.2) belongs to the family $\Sigma^{*}(a, b)$, and if $F^{*}(z)=(1 / z)+a_{n}^{*} z^{n}+\ldots$, then $\left|a_{n}^{*}\right|=(a-b) /(n+1)$ for $n=0,1,2, \ldots$, then estimate (3.1) is sharp.

Corollary 1. If $F \in \Sigma^{*}(a, b)$, then

$$
\sum_{k=0}^{\infty}\left(\left(1-b^{2}\right) k^{2}+2(1-a b) k+\left(1-a^{2}\right)\right)\left|a_{k}\right|^{2} \leqslant(a-b)^{2} .
$$


In fact, by equality (3.4) and the condition $|\omega(z)|<1$, we have

$$
\sum_{k=0}^{\infty}\left|(k+1) a_{k} z^{k+1}\right|^{2}<\left|a-b+\sum_{k=0}^{\infty}(a+k b) a_{k} z^{k}\right|^{2}
$$

thus

$$
\int_{0}^{2 \pi}\left|\sum_{k=0}^{\infty}(k+1) a_{k} r^{k+1} e^{i(k+1) r}\right|^{2} d t \leqslant \int_{0}^{2 \pi}\left|a-b+\sum_{k=0}^{\infty}(a+k b) a_{k} r^{k} e^{i(k+1) r}\right|^{2} d t .
$$

Hence, by integrating and passing to the limit as $r$ tends to 1, we obtain (3.8).

Corollary 2. By putting $a=1-\alpha-\alpha m, b=-m$ in (3.1) and (3.8) we get the corresponding result obtained in [4], and by letting $b=-a, 0<a \leqslant 1$, we get the result of [8]; finally, we remark that if $b=-1$ and $a=1-2 \alpha$, then we get the result obtained in [6].

\section{References}

[1] J. Clunie, On meromorphic schlicht, J. London Math. Soc. 34 (1959), p. 215-216.

[2] W. Janowski, Some extremal problems for certain families of analytic functions I, Ann. Polon. Math. 28 (1973), p. 297-326.

[3] -, Extremal problems for a family of functions with positive real part and for some related families, ibidem 23 (1970), p. 159-177.

[4] J. Kaczmarski, On the coefficients of some classes of starlike functions, Bull, Acad. Polon. Sci. 17 (1969), p. 495-501.

[5] W. Plaskota, Limitation des coefficients dans une famille de fonctions holomorphes dans le cercle $|z|<1$, Ann. Polon. Math. 24 (1970), p. 65-70.

[6] Ch. Pomerenke, On meromorphic starlike functions, Pacific J. Math. 13 (1963), p. 221-235.

[7] M. S. Robertson, On the theory of univalent functions, Ann. of Math. 37 (1936), p. 169- 185.

[8] Z. Wieczorek, On the coefficients of starlike functions of some classes, Comment. Math. 18 (1974), p. 113-119. 